\section{FedUni ResearchOnline}

\section{https://researchonline.federation.edu.au}

Copyright Notice

This is the peer-reviewed version of the following article:

Hewawasam, H.s \& Ibrahim, Yousef \& Kahandawa Appuhamillage, Gayan \& Choudhury, Tanveer. (2019). Agoraphilic Navigation Algorithm in Dynamic Environment with and without Prediction of Moving Objects Location. 5179-5185.

Which has been published in final form at:

https://doi.org/10.1109/IECON.2019.8927145

Copyright $\odot 2019$ IEEE. Personal use of this material is permitted. Permission from IEEE must be obtained for all other uses, in any current or future media, including reprinting/republishing this material for advertising or promotional purposes, creating new collective works, for resale or redistribution to servers or lists, or reuse of any copyrighted component of this work in other works. 


\title{
Agoraphilic Navigation Algorithm in Dynamic Environment with and without Prediction of Moving Objects Location
}

\author{
H.S. Hewawasam*, M. Yousef Ibrahim ${ }^{\dagger}$, Gayan Kahandawa ${ }^{\ddagger}$ and T. A. Choudhury ${ }^{\S}$ \\ School of Science, Engineering and Information Technology \\ Federation University Australia, Churchill \\ Vic. 3842, Australia \\ Email: *h.hewawasam@federation.edu.au, ${ }^{\dagger}$ yousef.ibrahim@federation.edu.au, ${ }^{\ddagger}$ g.appuhamillage@ federation.edu.au, \\ \$t.choudhury@federation.edu.au
}

\begin{abstract}
This paper presents a summary of research conducted in performance improvement of Agoraphilic Navigation Algorithm under Dynamic Environment (ANADE). The ANADE is an optimistic navigation algorithm which is capable of navigating robots in static as well as in unknown dynamic environments. ANADE has been successfully extended the capacity of original Agoraphilic algorithm for static environment. However, it could identify that ANADE takes costly decisions when it is used in complex dynamic environments. The proposed algorithm in this paper has been successfully enhanced the performance of ANADE in terms of safe travel, speed variation, path length and travel time.

The proposed algorithm uses a prediction methodology to estimate future growing free space passages which can be used for safe navigation of the robot. With motion prediction of moving objects, new set of future driving forces were developed. These forces has been combined with present driving force for safe and efficient navigation.

Furthermore, the performances of proposed algorithm (Agoraphilic algorithm with prediction) was compared and benchedmarked with ANADE (Without predication) under similar environment conditions. Form the investigation results, it was observed that the proposed algorithm extends the effective decision making ability in a complex navigation environment. Moreover, the proposed algorithm navigated the robot in a shorter and quicker path with smooth speed variations.

Index Terms-Agoraphilic algorithm, prediction, mobile robot navigation, artificial potential Field, free Space, dynamically cluttered environment
\end{abstract}

\section{INTRODUCTION}

The Agoraphlic algorithm is a local navigation algorithm used for mobile robots' navigation. The algorithm searches for space (solutions) to follow rather than for obstacles (problems) to avoid. Therefore, the Agoraphlic algorithm can be identified as an optimistic algorithm combined with a special force shaping mechanism. The Artificial Potential Field (APF) concept was introduced by Khatib [1]. Although the APF method is relatively simple, there are several well-documented problems associated with APF [2], [3], [4]. Such as,

1) When there is an obstacle close to the goal robot can't reach the goal.
2) When attraction and repulsive forcers are equal robot finds local minima.

3) In dynamic environments traditional approaches fail to implement the navigation task.

4) Robot exhibits oscillatory motions when moving in a narrow corridor.

5) Robot fails to go through narrow openings.

Those inherent challengers associated with APF have motivated researchers to address these issues and improve the basic APF algorithm. As a result, some algorithms were developed in last few years to address those issues. Sfeir et.al. [5] proposed an algorithm to minimize oscillations when the goal is close to obstacles. Using a virtual target strategy, W. Siming [3] developed an algorithm to improve APF by eliminating the target inaccessibility problem. Methodologies capable of navigating robots in dynamic environments while minimizing drawbacks of APF were discussed in [6], [7] and [8]. An improved APF based regression search methodology which was developed by G. Li, A. Yamashita can obtain sub-optimal/optimal path efficiently without local minima and oscillations in known environments [9]. Other methodologies have been developed to minimize limitations of APF algorithm such as Vector Field Histogram technique [10], Rulebased methods, Rules Learning Techniques [11] and Dynamic window approach [12].

However, all the above-mentioned methodologies were relying on the basic concept of APF by using attractive forces from goal as well as repulsive forces from obstacles in order to drive the robot. On the other hand Agoraphilic algorithm uses single force to drive the robot which is always an attractive force created by the space. This concept has allowed the original Agoraphilic algorithm to overcome limitations of traditional APF algorithm. However, the current Agoraphilic algorithm can't be used in a dynamic environments.

The authors' previous work was involved in eliminating this limitation of Agoraphilic algorithm [4]. This paper presents the new additional improvements done to the Agoraphlic algorithm. This improvement achieved by predicting future 
locations of moving objects in dynamically cluttered environments. These improvements have allowed the robot to navigate in dynamic environments more efficiently in terms of path length, speed and decision making compared with the previous methodologies [4].

The paper is organized in the following order: Section II describes Algoraphilic algorithm for dynamic environment. Section III presents simulation results for various cases. Section IV provides a comparative study between improved Algoraphilic algorithm for dynamic environments and the basic Algoraphilic algorithm for dynamic environments using the generated results. Section $\mathrm{V}$ presents discussion of the obtained results and conclusions.

\section{Agoraphilic Navigation Algorithm Under DYNAMIC ENVIRONMENT}

This section will introduce the Agoraphilic algorithm in a dynamic environment against the backdrop of the previous work of this Algorithm in a static environment.

\section{A. Agoraphlic algorithm for static environment (Original Ago- raphilic Algorithm)}

The Agoraphilic algorithm differs from APF methodology as it uses a Free-Space Force (FSF) concept [13], [14]. This force is an attractive force which pulls the robot through the free space to the goal. To guide the robot to the desired goal FSFs are shaped using a force-shaping function [15].

The original Agoraphilic algorithm which is designed for static environment consists of four basic stages:

1) Creation of Free-Space Histogram (FSH).

2) Calculation of free-space forces.

3) Generation of the force shaping coefficients.

4) Summation of scaled force vectors.

\section{B. Agoraphlic navigation algorithm under dynamic environ- ment with prediction}

The new algorithm developed for dynamic environment follows the basic concepts of the original algorithm with additional improvements to cope with dynamics obstacles. In the new algorithm, the basic stages are modified and used along with dynamic tracking, prediction, current global map generation, and future global map generation as listed in the following steps:

1) Object tracking and current global map generation.

2) Prediction of future locations of objects and future global map generation.

3) Creation of Free-Space Histogram (FSH).

4) Calculation of free-space forces.

5) Generation of the force shaping coefficients.

6) Summation of scaled force vectors.

Each of the above stage is briefly described below.
1) Object tracking and Current Global Map (CGM) generation: Object tracking and current global map generation stage is used to estimate the current locations of moving objects and generate the current global map. Current global map (CGM) is used in FSH creation stage.

The current positions of moving objects cannot be accurately tracked using only sensory systems because of the sensors' noise [16]. A Kalman Filter (KF) based tracking algorithm is used to track and estimate current stats (location and velocity) of moving objects. The tracking algorithm uses two basic models, process model and measurement model.

The process model is developed considering moving objects as slow maneuvering objects by maintaining an adequate sampling frequency [17]. This model is used to pre-estimate the states of moving objects. Due to the variations of accelerations of the moving objects the pre-estimations are usually noisy. This is further compounded with inaccurate states measurements due to sensory noise. The KF is used to combine preestimated states and measured states to estimate the current states of moving objects with increased accuracy [16].

The estimated locations of the moving objects and locations of static objects are transferred to a global access system to get the current global map. Current global map is then used in creating the FSH.

2) Prediction of future locations of objects and Future Global Map (FGM) generation: The prediction of moving obstacles' future location is very important for mobile robots navigation in dynamic environment. Therefore, at this stage a prediction methodology is used to predict future locations of moving objects and develop global maps for future samples (if the prediction is done till $t+n$ sample, there will be $n-1$ future global maps).

Using a prediction model (Eq. 1) future states (location and velocity) are predicted. Predicted states are corrected in next sample and new predictions will be done.

Future global map for $\mathrm{N}^{\text {th }}$ sample is generated by combining locations of moving objects in $\mathrm{N}^{t h}$ sample with locations of static objects and transferring the object positions to a global coordinates system. These future global maps are also used for the FSH creation.

$$
\begin{aligned}
{\left[\begin{array}{c}
x(t+n) \\
y(t+n) \\
d x(t+n) / d t \\
d y(t+n) / d t
\end{array}\right]=} & {\left[\begin{array}{cccc}
1 & 0 & n T & 0 \\
0 & 1 & 0 & n T \\
0 & 0 & 1 & 0 \\
0 & 0 & 0 & 1
\end{array}\right]\left[\begin{array}{c}
x(t) \\
y(t) \\
d x(t) / d t \\
d y(t) / d t
\end{array}\right]+} \\
& {\left[\begin{array}{c}
1 / 2(n T)^{2} \\
1 / 2(n T)^{2} \\
n T \\
n T
\end{array}\right] u(t) }
\end{aligned}
$$

$\begin{array}{rll}x(t) & \stackrel{\text { def }}{=} & \text { position in x direction } \\ y(t) & \stackrel{\text { def }}{=} \text { position in y direction } \\ T & \stackrel{\text { def }}{=} & \text { sample period } \\ n & \stackrel{\text { def }}{=} & \text { sample number }\end{array}$


3) Creation of Free-Space Histogram (FSH): Free-Space Histogram is a robot-centered polar map. This represents a distance profile of a global map. FSHs are generated for the CGM and for all FGMs per sample. FSHs are used to generate FSFs [15].

Using the information from the corresponding global map, occupied cells of the polar map are determined. A predetermined safety boundary is applied to occupied cell using an enlargement technique. This technique is similar to the technique used in Ulrich and Borenstein's VFH+ algorithm [18]. The surrounding of the robot is divided into $\mathrm{K}$ neighboring sectors $(1,2, \ldots, \mathrm{k}, \ldots \mathrm{K})$. Minimum distance to a selected point on safety boundary from the robot is calculated $\left(\mathrm{d}_{k}\right)$ for each sector.

The distance profile which contains $\mathrm{d}_{k}$ information creates a robot-centered polar map

This process is applied for all created FGMs and for the CGM in a sample.

4) Calculation of free-space forces: Distance profile in FSH is used in this section to create the Free-Space Forces (FSFs).

For each sector ' $\mathrm{k}$ ', an initial force $\left(\mathrm{F}_{k}\right)$ is created. Initial force in a sector is directly proportional to the square of the normalized distance correspondent to the sector, Eq. 2. When generating initial force $\left(\mathrm{F}_{k}\right)$, if the distance in FSH corresponding to that sector $\left(\mathrm{d}_{k}\right)$ is greater than pre-determined dmax the initial force of the sector is taken as uk [4]. These initial forces are known as FSFs. This process is applied for all the FGMs and for CGM in each sample and creates an individual set of FSFs for each map.

$$
\overrightarrow{F_{k}}=\left(d_{k} / d_{\max }\right)^{2} \cdot \overrightarrow{u_{k}}
$$

$$
\begin{aligned}
& \text { Where: } \\
& \overrightarrow{u_{k}} \stackrel{\text { def }}{=}\left[\cos \left(\theta_{k}\right) \sin \left(\theta_{k}\right)\right]
\end{aligned}
$$

5) Generation of the force shaping coefficients: The sets of FSFs generated for each map need to be remodified with a weighting system to focus the forces to the goal. This freespace force modification is known as force shaping.

In force shaping mechanism, FSFs towards the goal get higher weight with respect to FSFs pointing away from the goal [15]. Force shaping coefficient $(\delta)$ for initial force $\mathrm{F}_{k}$ is decided by the Eq. 3. Force shaping coefficient gets its maximum value of "one" when the corresponding $\mathrm{F}_{k}$ is aligned with the goal. When $\mathrm{F}_{k}$ does not make a positive force towards the goal or when it is perpendicular to the goal the corresponding $\delta$ becomes zero. Scaled sector forces are generated by scaling the initial sector forces using force shaping coefficients.

The force shaping step is applied to all sets of FSFs generated for all the FGMs and for the CGM in a sample.

$$
\delta(\theta)= \begin{cases}\frac{1}{90} \theta+1, & \text { if }-90 \leq \theta \leq 0 \\ \frac{-1}{90} \theta+1, & \text { if } 0 \leq \theta \leq 90 \\ \frac{1}{90} \theta-3, & \text { if } 270 \leq \theta \leq 360 \\ \frac{-1}{90} \theta-3, & \text { if }-270 \leq \theta \leq 360 \\ 0, & \text { otherwise }\end{cases}
$$

6) Summation of scaled force vectors: In this stage the robot's driving force is generated. Resultant force $\left(\mathrm{F}_{r}\right)$ for each set of shaped FSFs (Scaled sector forces) are calculated by taking the vector summation. This generates a resultant force for CGM $\left(\mathrm{F}_{r}(\mathrm{t})\right)$ and a resultant force for each FGMs $\left(\mathrm{F}_{r}(\mathrm{t}+1), \mathrm{F}_{r}(\mathrm{t}+2), \ldots, \mathrm{F}_{r}(\mathrm{t}+\mathrm{n})\right)$. Then the vector summation of weighted resultant forces $\left(\mathrm{F}_{r}\right)$ are applied to calculate the final robot driving force $\mathrm{F}_{t}$.

\section{Simulation Results}

Experiments were conducted to investigate and assess the effect of the obstacles location prediction algorithm on the navigation characteristics of a mobile robot. To study this effect, four navigation experiments were conducted without the prediction algorithm and repeated with the prediction Algorithm. All the individual experiments were performed exactly under same conditions for both algorithms (with obstacles motion prediction and without the obstacles motion prediction). The results and four examples are discussed below.

\section{A. Experiment 1: Navigation in Static Environment}

In this experiment algorithms were tested in an environment which had only four static obstacles. Robot's starting position coordinates $(-950,-900)$, goal location coordinates were $(-200$, 950).

Key Findings of this experiments:

- Both the algorithms have taken decisions in the same way in most of the cases.

- Time taken to reach the goal was almost the same for both.

- Agoraphilic algorithm with prediction has taken a slightly shorter path.

B. Experiment 2: Navigation in Dynamic Environment with one moving object

Conditions of this experiment:

- Goal location: (-200, 950)

- Robots stating point: $(-250,-700)$

- Number of static objects: 3

- Number of moving objects: 1 (MO)

TABLE I

INITIAL STATES OF MOVING OBJECT IN EXPERIMENT2

\begin{tabular}{|c|c|c|}
\hline Moving Object & Position (x,y) / cm & Velocity (x,y) / cm/s \\
\hline MO & $(250,200)$ & $(-5.0,1.9)$ \\
\hline
\end{tabular}

Robot's paths for two independent simulation runs with the above mentioned conditions that shown in Fig. 1. It can be 


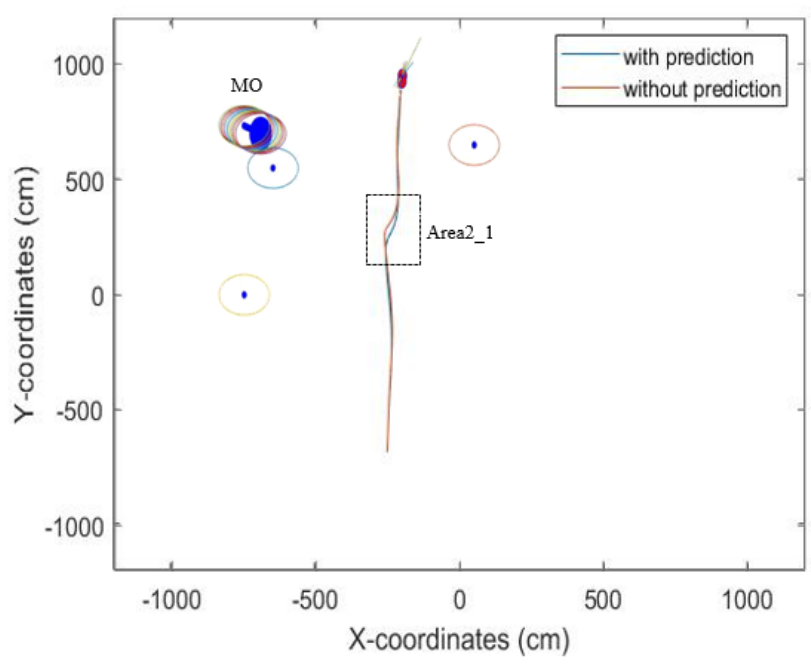

Fig. 1. Comparison of navigation paths in dynamic environment with one moving object

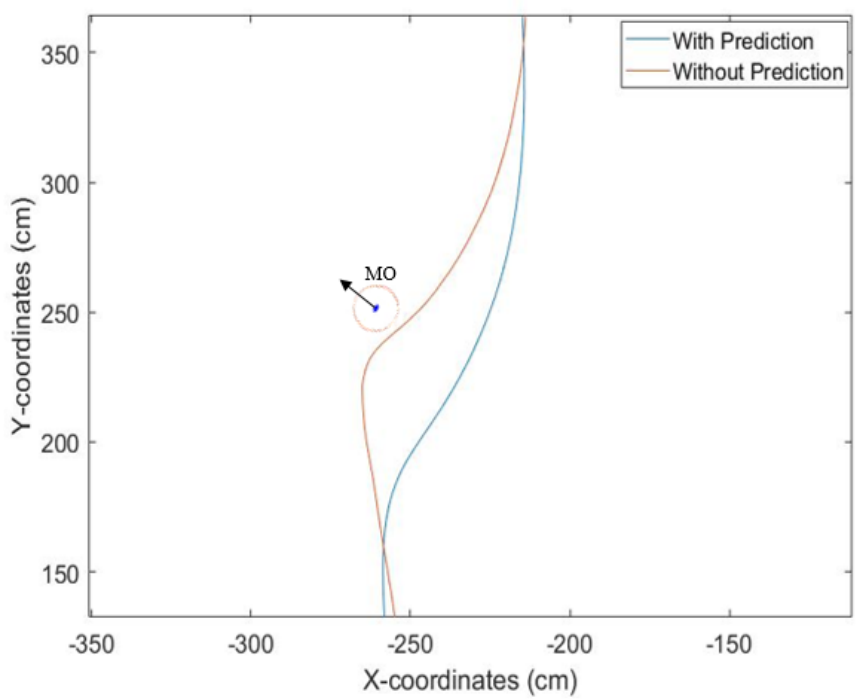

Fig. 2. Comparison of navigation paths in dynamic environment with one moving object zoomed Area2_1

observed in this experiment that without prediction the robot moves very close to the moving object "MO" and takes a sharp turn to avoid the collision (Fig. 1). In this case robot had to decelerate quickly and change its direction, Fig. 3. Furthermore, the robot has travelled around $1726 \mathrm{~cm}$ to reach the goal and it has taken around 170s to complete the task.

On the other hand, robot which had the Agoraphilic algorithm with prediction (of moving object's future location) used a different path. Taking the advantage of prediction algorithm, the robot started moving towards $(+\mathrm{x},+\mathrm{y})$ direction (MO moved to $(-\mathrm{x},+\mathrm{y})$ direction) before getting very close to MO. This has allowed the robot to avoid the collation without taking any sharp turns (Fig. 2). Also in this case robot has

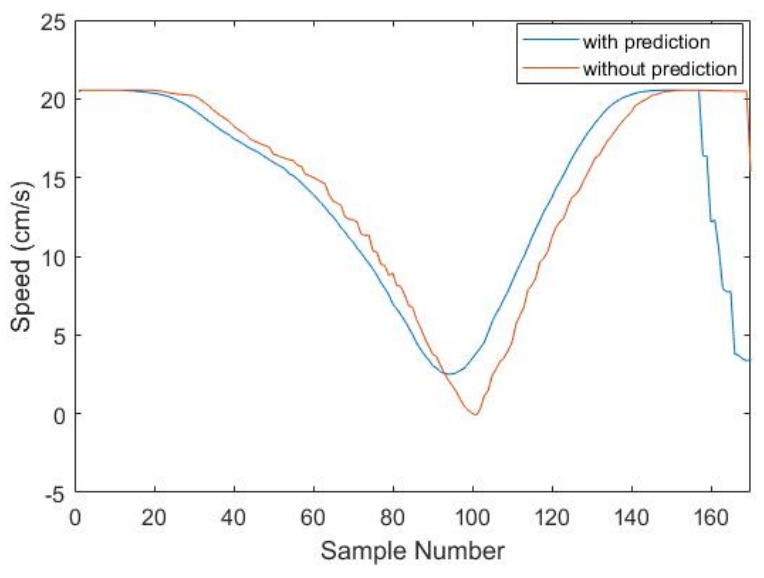

Fig. 3. Comparison of speed variations in dynamic environment with one moving object

decelerated and accelerated in a smoother way as shown in Fig. 3. Total traveled length of the robot was around $1662 \mathrm{~cm}$. Total time taken was around 160s.

Key findings of this experiment:

- Prediction has allowed the robot to make prior decisions when it engages with moving obstacles.

- Prediction has avoided the robot from getting sharp turns (smoother path).

- Prediction has reduced the path length when avoiding moving objects.

- Prediction has improved the smoothness of speed variations.

C. Experiment 3: Dynamic environment with multipul moving objects

Conditions of this experiment:

- Goal location: $(-200,950)$

- Robots stating point: $(-250,-700)$

- Number of static objects: 3

- Number of moving objects: 3 (MO1, MO2, MO3)

TABLE II

INITIAL STATES OF MOVING OBJECT IN EXPERIMENT3

\begin{tabular}{|c|c|c|}
\hline Moving Object & Position $(\mathbf{x , y}) / \mathbf{~ c m}$ & Velocity $(\mathbf{x , y}) / \mathbf{~ c m} / \mathbf{s}$ \\
\hline MO1 & $(-500,-200)$ & $(1.0,0.6)$ \\
\hline MO2 & $(-550,100)$ & $(1.9,0.4)$ \\
\hline MO3 & $(250,200)$ & $(-5.0,1.9)$ \\
\hline
\end{tabular}

Key Findings of this experiment:

- In a dynamic environment with multiple moving objects, prediction methodology has significantly influenced in reducing the path length.

- Prediction has allowed the robot to reach the goal quicker by maintain smooth speed variations and taking shorter paths.

- Prediction has allowed the robot to make prior decisions when it engages with moving obstacles.

- Prediction has avoided the robot from getting sharp turns. 
D. Experiment 4: Dynamic environment with cluttered moving objects

Conditions of this experiment:

- Goal location: (-200, 950)

- Robots stating point: $(-150,-1000)$

- Number of static objects: 3

- Number of moving objects: 3 (MO1, MO2, MO3)

TABLE III

INITIAL STATES OF MOVING OBJECT IN EXPERIMENT4

\begin{tabular}{|c|c|c|}
\hline Moving Object & Position $(\mathbf{x}, \mathbf{y}) / \mathbf{c m}$ & Velocity $(\mathbf{x}, \mathbf{y}) / \mathbf{c m} / \mathbf{s}$ \\
\hline MO1 & $(-500,-200)$ & $(1.5,1.5)$ \\
\hline MO2 & $(-550,100)$ & $(4.3,0.4)$ \\
\hline MO3 & $(250,350)$ & $(-2.0,-0.8)$ \\
\hline
\end{tabular}

Robot's paths for two independent simulation runs with above mentioned conditions are shown in Fig. 4. This experiment was conducted to test the ability of algorithms to work in dynamically cluttered environments with complex situations (multiple obstacles interrupting the path of the robot simultaneously). In this case robots' motion is influenced by three moving objects (MO1, MO2, MO3) at the same time.

Initially (at around $\mathrm{t}=75 \mathrm{~s}$ ) both the algorithms has pushed the robot towards Passage_1, Fig. 4. At around $t=110 \mathrm{~s}$, free space of Passage_1 start decreasing and it has started making a new passage (Passage_2), Fig. 5. However, at this time instant Passage_1 has more free space than Passage_2. Based on current available free space, the algorithm without prediction has allowed the robot to move further towards Passage_1. As a result of this decision robot trapped in Passage_1 and failed to avoid the collision with MO3.

On the other hand, algorithm with prediction has reduced the velocity of the robot at sample 110 . Then turned the robot towards Passage_2 although it has less free space compared to Passage_1. The reason behind this decision is algorithm with prediction looks for free space which will be created in future. Consequently, the robot could avoid the trap in Passage_1 and could safely move through the increasing Passage_2.

Key Findings:

- In a dynamic cluttered environments the prediction methodology guide the robot away from traps.

- Prediction has allowed the robot to identify future possible free spaces.

- Prediction has allowed the robot to navigate safely in complex environments.

\section{COMParision of Agoraphilic Algorithm With PREDICTION AND WITHOUT PREDICTION}

In all conducted experiments except the fourth experiment both the algorithms (with and without obstacles motion prediction) were able to find collision free paths to reach the goal. In the fourth experiment, the algorithm without prediction collided with an obstacle.

When the first experiment which was conducted in a static environment is considered, both the algorithms has performed

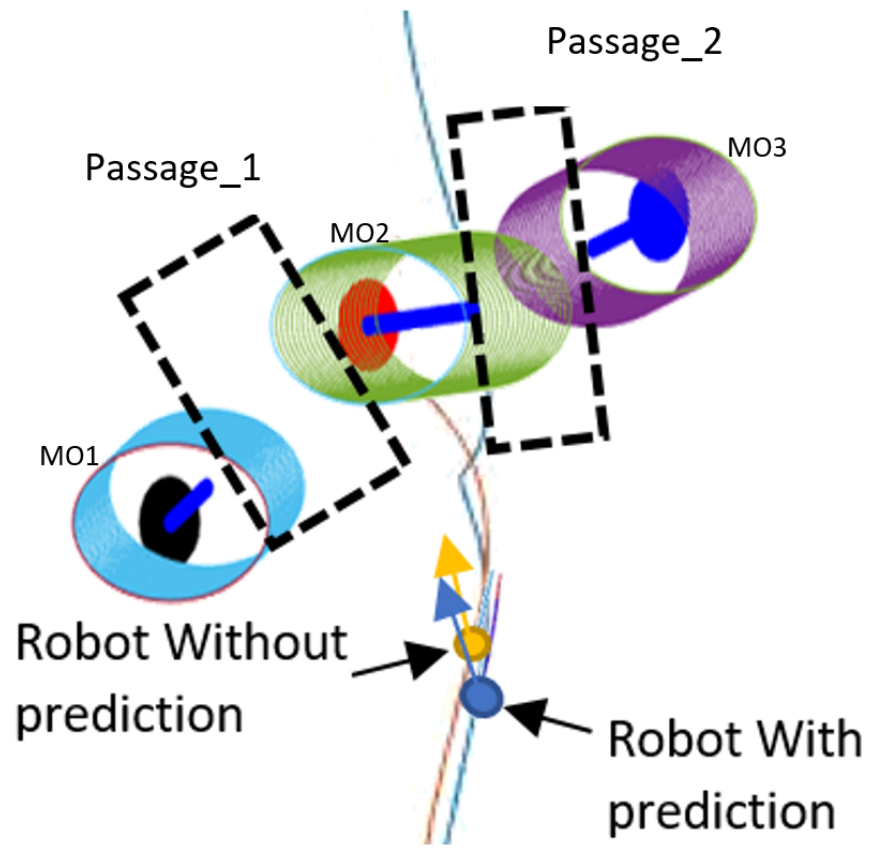

Fig. 4. Robot's surrounding at around $t=75 \mathrm{~s}$ with dynamically cluttered environment

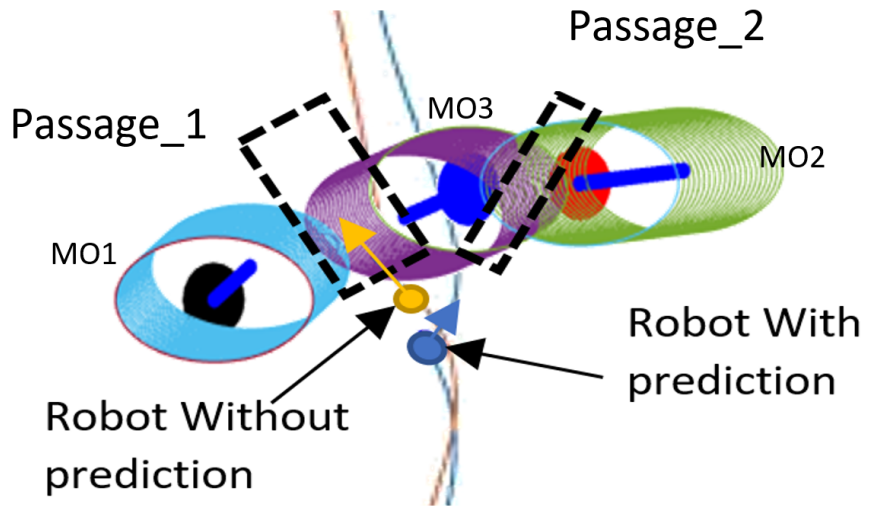

Fig. 5. Robot's surrounding at around $\mathrm{t}=110 \mathrm{~s}$ with dynamically cluttered environment

almost in the same way. Path length with prediction is $5.8 \%$ less than without prediction. To reach the goal both the algorithms took almost same time. There was no significant differences in decision making between two algorithms in static environment.

However, during the second experiment which was conducted in a dynamic environment, the Agoraphilic navigation algorithm exhibited some advantages of using the obstacle motion prediction algorithm. In this case the algorithm with prediction has reached the goal 10s $(6.3 \%)$ earlier. Furthermore, it has used a shorter and smoother path with smooth speed variations. In this experiment when robot engaged with MO1 Agoraphilic algorithm without prediction has driven the robot very close to MO1 and then has taken a sharp turn to 
prevent a collision (Area2_1). This has created a longer path for the robot. Moreover, during this period robot has to reduce its speed rapidly and stopped (Fig. 3). On the other hand robot which was controlled by Agoraphilic algorithm with prediction has identified that robot cannot pass the MO1 by moving to the same direction as MO1. Then it has started moving to - $\mathrm{x}$ direction before it get close to MO1 (Area2_1). This has allowed the robot to find a smoother and shorter collision free path. Furthermore, the robot has stared reducing its speed before getting too close to MO1 which has prevented the robot from a rapid deceleration. In this period robots speed variation was smoother compared to algorithm without prediction.

The third experiment presents in this paper was also conducted in a dynamic environment but with many moving objects. This experiment highlighted the many advantages of using Agoraphlic algorithm with prediction (Table VI) for local navigation tasks. By comparing the two algorithms in this experiment, it was found that the algorithm with prediction uses a $32 \%$ shorter and $12 \%$ quicker path compared to algorithm without obstacle motion prediction. During the process robot maneuvered from algorithm with prediction has never stopped and has maintained smooth speed variations.

The presented fourth experiment was conducted in a dynamically cluttered complex environment. Form this experiment it could observed that algorithm with prediction maneuvered the robot safely through complex environments compared to algorithm without prediction. Furthermore, this experiment showed the importance of estimating future free spaces to avoid robots getting into traps.

TABLE IV

COMPARATIVE RESULTS FOR EXPERIMENT 1: STATIC ENVIRONMENT

\begin{tabular}{|c|c|c|}
\hline Parameters & Without Prediction & With Prediction \\
\hline Path Length $(\mathrm{cm})$ & 3398 & 3210 \\
\hline Time taken $(\mathrm{s})$ & 221 & 220 \\
\hline Avg. speed $(\mathrm{cm} / \mathrm{s})$ & 15 & 15 \\
\hline Minimum speed $(\mathrm{cm} / \mathrm{s})$ & 5.3 & 6.4 \\
\hline Max speed $(\mathrm{cm} / \mathrm{s})$ & 21.5 & 21.5 \\
\hline
\end{tabular}

TABLE V

COMPARATIVE RESULTS FOR EXPERIMENT 2: DYNAMIC ENVIRONMENT WITH ONE MOVING OBJECT

\begin{tabular}{|c|c|c|}
\hline Parameters & Without Prediction & With Prediction \\
\hline Path Length $(\mathrm{cm})$ & 1726 & 1662 \\
\hline Time taken $(\mathrm{s})$ & 170 & 160 \\
\hline Avg. speed $(\mathrm{cm} / \mathrm{s})$ & 10 & 10 \\
\hline Minimum speed $(\mathrm{cm} / \mathrm{s})$ & 0 & 2.4 \\
\hline Max speed $(\mathrm{cm} / \mathrm{s})$ & 20.5 & 20.5 \\
\hline
\end{tabular}

\section{CONCLUSION}

This paper presents a summary of research conducted on the performance improvements of the Agoraphilic Navigation Algorithm under Dynamic Environment (ANADE ). The proposed algorithm uses obstacle motion prediction methodology to estimate and identify the growing free space passages. This
TABLE VI

COMPARATIVE RESULTS FOR EXPERIMENT 3: DYNAMIC ENVIRONMENT WITH MANY MOVING OBJECTS

\begin{tabular}{|c|c|c|}
\hline Parameters & Without Prediction & With Prediction \\
\hline Path Length $(\mathrm{cm})$ & 2456 & 1666 \\
\hline Time taken $(\mathrm{s})$ & 185 & 165 \\
\hline Avg. speed $(\mathrm{cm} / \mathrm{s})$ & 13 & 10 \\
\hline Minimum speed $(\mathrm{cm} / \mathrm{s})$ & 0 & 5.7 \\
\hline Max speed $(\mathrm{cm} / \mathrm{s})$ & 23.5 & 23.5 \\
\hline
\end{tabular}

helps the robots for safe and efficient navigation. Tracking algorithm along with prediction methodology create Current and Future Global Maps (CGM and FGMs) for the each iteration in navigation process. Free-Space Forces (FSFs) are developed based on CGM and FGMs. Consequently, the FSFs are used to generate the current single driving force of the robot. This approach enables the robot's instantaneous driving force to incorporate the predicted future free space as well.

The investigating results clearly shown the proposed algorithm has improved the performance of Algoraphilic algorithm in terms of decision making, speed controlling, path length and travel time.

Moreover, this paper presented a comparative analysis of Agoraphilic algorithm with prediction and without prediction. The conducted study proved that prediction of obstacle's motion locations methodology is capable of detecting future free space passages which do not exist in instantaneous mapping. Navigation algorithm with prediction uses predicted passages for the safe current navigation of the robot in an environment with multiple moving objects. In contrast, the research results has shown that Agoraphilic algorithm without prediction has failed to navigate robot in these complex environments without collusions. Furthermore, it was also observed that algorithm with prediction was able to navigate the robot in $38 \%$ shorter distance and $12 \%$ faster path with smooth speed variations in an unknown dynamic environment. Effectiveness of future location prediction of moving objects increases when number of moving objects and the complexity of the environment increase.

\section{REFERENCES}

[1] O. Khatib, "Real-time obstacle avoidance for manipulators and mobile robots," in Proceedings. 1985 IEEE International Conference on Robotics and Automation, vol. 2, March 1985, pp. 500-505.

[2] M. Y. Ibrahim and A. Fernandes, "Study on mobile robot navigation techniques," in 2004 IEEE International Conference on Industrial Technology, 2004. IEEE ICIT '04., vol. 1, Dec 2004, pp. 230-236 Vol. 1.

[3] W. Siming, Z. Tiantian, and L. Weijie, "Mobile robot path planning based on improved artificial potential field method," in 2018 IEEE International Conference of Intelligent Robotic and Control Engineering (IRCE), Aug 2018, pp. 29-33.

[4] H. S. Hewawasam, M. Y. Ibrahim, G. Kahandawa, and T. A. Choudhury, "Development and bench-marking of agoraphilic navigation algorithm in dynamic environment," in 2019 IEEE International Symposium on Industrial Electronics, June 2019.

[5] J. Sfeir, M. Saad, and H. Saliah-Hassane, "An improved artificial potential field approach to real-time mobile robot path planning in an unknown environment," in 2011 IEEE International Symposium on Robotic and Sensors Environments (ROSE), Sep. 2011, pp. 208-213. 
[6] O. Montiel, U. Orozco-Rosas, and R. Sepúlveda, "Path planning for mobile robots using bacterial potential field for avoiding static and dynamic obstacles," Expert Systems with Applications, vol. 42, no. 12, pp. 5177 - 5191, 2015. [Online]. Available: http://www.sciencedirect.com/science/article/pii/S0957417415001402

[7] B. Kovács, G. Szayer, F. Tajti, M. Burdelis, and P. Korondi, "A novel potential field method for path planning of mobile robots by adapting animal motion attributes," Robotics and Autonomous Systems, vol. 82, pp. 24 - 34, 2016. [Online]. Available: http://www.sciencedirect.com/science/article/pii/S0921889016302159

[8] M. A. K. Jaradat, M. H. Garibeh, and E. A. Feilat, "Autonomous mobile robot dynamic motion planning using hybrid fuzzy potential field," Soft Computing, vol. 16, no. 1, pp. 153-164, Jan 2012. [Online]. Available: https://doi.org/10.1007/s00500-011-0742-z

[9] G. Li, A. Yamashita, H. Asama, and Y. Tamura, "An efficient improved artificial potential field based regression search method for robot path planning," in 2012 IEEE International Conference on Mechatronics and Automation, Aug 2012, pp. 1227-1232.

[10] J. Borenstein and Y. Koren, "The vector field histogram-fast obstacle avoidance for mobile robots," IEEE Transactions on Robotics and Automation, vol. 7, no. 3, pp. 278-288, June 1991.

[11] B. Babic, N. Nesic, and Z. Miljkovic, "A review of automated feature recognition with rule-based pattern recognition," Computers in Industry, vol. 59, no. 4, pp. 321 - 337, 2008. [Online]. Available: http://www.sciencedirect.com/science/article/pii/S0166361507001327

[12] P. Ogren and N. E. Leonard, "A convergent dynamic window approach to obstacle avoidance," IEEE Transactions on Robotics, vol. 21, no. 2, pp. 188-195, April 2005.

[13] M. Y. Ibrahim and L. McFetridge, "The agoraphilic algorithm: a new optimistic approach for mobile robot navigation," in 2001 IEEE/ASME International Conference on Advanced Intelligent Mechatronics. Proceedings (Cat. No.01TH8556), vol. 2, July 2001, pp. 1334-1339 vol.2.

[14] L. McFetridge and M. Y. Ibrahim, "Behavior fusion via free-space force shaping," in IEEE International Conference on Industrial Technology, 2003, vol. 2, Dec 2003, pp. 818-823 Vol.2.

[15] L. McFetridge and M. Ibrahim, "A new methodology of mobile robot navigation: The agoraphilic algorithm," Robotics and Computer-Integrated Manufacturing, vol. 25, no. 3, pp. 545 - 551, 2009. [Online]. Available: http://www.sciencedirect.com/science/article/pii/S0736584508000495

[16] H. S. Hewawasam, M. Y. Ibrahim, G. Kahandawa, and T. A. Choudhury, "Comparative study on object tracking algorithms for mobile robot navigation in gps-denied environment," in 2019 IEEE International Conference on Industrial Technology, Feb 2019.

[17] A. Elnagar, "Prediction of moving objects in dynamic environments using kalman filters," in Proceedings 2001 IEEE International Symposium on Computational Intelligence in Robotics and Automation (Cat. No.01EX515), 2001, Conference Proceedings, pp. 414-419.

[18] I. Ulrich and J. Borenstein, "Vfh+: reliable obstacle avoidance for fast mobile robots," in Proceedings. 1998 IEEE International Conference on Robotics and Automation (Cat. No.98CH36146), vol. 2, May 1998, pp. 1572-1577 vol.2. 